\title{
Phosphatidylinositol 3-kinase and trypsin activation in pancreatitis
}

Commentary

See related article, pages $1387-1394$

\begin{abstract}
Craig Logsdon
University of Michigan, Department of Physiology, Box 0622, 7710 Medical Science Building II, Ann Arbor, Michigan 48109-0622. Phone: (203) 785-5279; Fax: (734) 936-8813; E-mail: clogsdon@umich.edu.
\end{abstract}

J. Clin. Invest. 108:1267-1268 (2001). DOI:10.1172/JCI200114272.

Acute pancreatitis is a complex inflammatory disease that begins with pancreatic injury and expands to involve multiple organs. Ultimately, the systemic rather than the local effects lead to the morbidity and mortality associated with the disease. However, in terms of prevention it is important to understand the initiation of the disease, which likely occurs within pancreatic acinar cells. The mechanisms that initiate acute pancreatitis have been the subject of a considerable body of research and are the focus of the article by Singh et al. (1) in the current issue of the JCI. This article provides interesting new insight into pancreatic acinar cell mechanisms responsible for the premature and inappropriate activation of trypsin.

\section{Cellular control of trypsin}

Trypsin is a serine protease that is produced and secreted by pancreatic acinar cells as a digestive enzyme. The idea that digestive enzymes play an important role in acute pancreatitis has existed for 100 years (2). Activation of trypsin is now believed to represent the linchpin of acute pancreatitis, and, indeed, trypsin is activated within pancreatic acinar cells early in the course of experimental acute pancreatitis (3). Therefore, there is much interest in understanding the cellular mechanisms involved in this activation process.

Inappropriate activation of digestive enzymes is normally prevented by several redundant mechanisms. First, many enzymes are produced as inactive precursors that are only activated in the small intestine, where enteropeptidase (also called enterokinase), an enterocyte plasma membrane protein, acts to cleave a small peptide, trypsin-associated peptide (TAP), from trypsinogen, the inactive form of trypsin. Trypsin then acts upon the other precursor digestive enzymes, leading to their functional activation. Second, the digestive enzymes are produced, sorted, and stored within membrane-bound vesicular compartments, thus isolating them from the rest of the cell. Third, a trypsin inhibitor is produced within acinar cells and packaged with the digestive enzymes within the secretory granule. Fourth, damaged cells are able to dispose of their digestive enzymes by a process of controlled lysosomal degradation. Within the lysosomes proteolytic enzymes, including the cathepsins, degrade and inactivate trypsin and the other digestive enzymes. Trypsin can also degrade

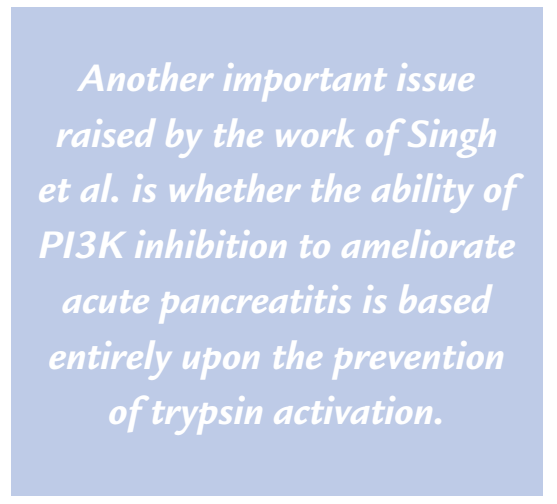

itself, a process that appears to play a protective role in the pancreas, as mutations in trypsin that prevent selfdegradation are responsible for a form of hereditary pancreatitis (4).

\section{Activation of trypsin \\ in the acinar cell}

Despite these various protective mechanisms, trypsin becomes activated within the pancreatic acinar cell during the pathogenesis of acute pancreatitis, probably within the lysosomes. These organelles contain cathepsin B and other proteases that can activate trypsin, and a number of cell fractionation studies have shown that zymogen granules and lysosomal markers colocalize following acinar cell damage (5). It has also been found that genetic deletion of Cathepsin $B$ reduces trypsin activation during caerulein-induced experimental acute pancreatitis (6). Moreover, both trypsin activity (7) and TAP (8) have been localized to the lysosomal compartment. Thus, there is strong evidence that trypsin can be activated within lysosomes by cathepsins.

Another important question involves the mechanisms responsible for the sorting of zymogen granules to lysosomes. Important new insight into this process comes from the study by Singh et al. (1). Normally the cell has mechanisms that carefully separate membrane compartments. For example, secretory granules traffic to the apical portion of the cell, whereas other smaller endocytotic ves icles may be targeted to the basal pole. The intricate process of membrane sorting is mediated by dynamic interactions of vesicles and organalles with the cytoskeleton. Recent studies of non-acinar cells (9) and yeast cells (10) show that secretory and endoctytic vesicle trafficking is regulated by phosphatidylinositol 3-kinase (PI3K). Wortmannin, a potent inhibitor of multiple classes of PI3K, blocks endosome fusion in vitro and impairs the transport of cathepsin D and internalized PDGF-R to the lysosome in vivo (11). In their present study, Singh et al. (1) show that wortmannin prevents trypsin activation in the pancreatic acinar cell, implying that PI3K activity is required for the fusion of lysosomes with secretory granules during caerulein hyperstimulation. Indeed, the authors provide evidence that zymogen and lysosomal markers fail to colocalize after wortmannin treatment. Surprisingly (in light of previous reports from other groups; see refs. 12-14), the authors 
found no evidence that caerulein treatment directly activates class I PI3K. Instead, the authors suggest that class III PI3Ks, which are believed to be constitutively active, may be involved. On this basis, the authors propose that basal, rather than stimulated, PI3K activity is required for the colocalization and fusion of lysosomes and zymogen granules, implying that PI3K activity may not be sufficient to induce trypsin activation.

\section{Pathogenic roles of PI3K}

Further research will be required to fully understand the function of PI3K in this response. It will also be important to discover the downstream mechanisms required to allow the active trypsin to escape the confines of the lysosome and damage the acinar cells. Trypsin activation within the lysosome would not be expected to cause cellular damage, and activation of trypsin within acinar cells has been shown to occur without causing acute pancreatitis when the secretagogue bombesin is utilized as a stimulant (15). Thus, the leakage of active trypsin out of lysosomes and into the cellular cytoplasm is a critical component of the development of acute pancreatitis.

Another important issue raised by the work of Singh et al. (1) is whether the ability of PI3K inhibition to ameliorate acute pancreatitis is based entirely upon the prevention of trypsin activation. In this study, PI3K inhibitors inhibited acinar cell trypsin activation and ameliorated the severity of pancreatitis, thus suggesting trypsin activation is both necessary and sufficient for the development of acute pancreatitis. However, the severity of acute pancreatitis depends upon the extent of the systemic inflammatory response. Much evidence now indicates that damaged acinar cells produce inflammatory mediators that recruit and activate leukocytes, and that these cells then amplify and extend the disease beyond the pancreas to involve the lungs and other organs. The expression of these inflammatory mediators has been linked to activation of the transcription factor NF- $\kappa B$, which plays a key role in many inflammatory diseases.
Recently it has been found that activation of NF-KB occurs within pancreatic acinar cells early in the course of experimental acute pancreatitis and is correlated with the expression of cytokines and chemokines (16-19). Thus, activation of NF- $\mathrm{KB}$ might be expected to induce an inflammatory response. However, in the present study, wortmannin did not block NF- $K B$ activation in acinar cells but prevented neutrophil infiltration of the pancreas, apparently arguing against a role for NF- $\kappa \mathrm{B}$ activity in the inflammatory response observed in acute pancreatitis. However, a potential explanation for the anti-inflammatory effects observed in the Singh et al. study is the well-documented ability of PI3K inhibitors to directly block inflammatory cell function (20). These inhibitors have potent effects to block both recruitment to sites of injury (21, 22) and activation (23) of inflammatory cells. Neutrophils are known to be critical for the severity of experimental acute pancreatitis $(24,25)$. Therefore, in the current study, it is not possible to determine to what extent wortmannin's effect on the severity of acute pancreatitis reflects its ability to inhibit trypsin activation within pancreatic acinar cells as opposed to its ability to inhibit inflammation directly. New approaches using treatments whose effects are restricted to the pancreas perhaps involving the targeted delivery or expression of a PI3K signaling inhibitor - may be required to resolve this uncertainty.

1. Singh, V.P., et al. 2001. Phosphatidylinositol-3 kinase-dependent activation of trypsinogen modulates the severity of acute pancreatitis. $J$. Clin. Invest. 108:1387-1394.

2. Chiari, H. 1896. Selbstverdauung des menschlichen pankreas. Z. Heilk. 17:69-96.

3. Lerch, M.M., and Gorelick, F.S. 2000. Early trypsinogen activation in acute pancreatitis. Med. Clin. North Am. 84:549-563.

4. Whitcomb, D.C. 1999. Hereditary pancreatitis: new insights into acute and chronic pancreatitis. Gut. 45:317-322.

5. Saluja, A., et al. 1987. Subcellular redistribution of lysosomal enzymes during caerulein-induced pancreatitis. Am. J. Physiol. 253:G508-G516.

6. Halangk, W., et al. 2000. Role of cathepsin B in intracellular trypsinogen activation and the onset of acute pancreatitis. J. Clin. Invest. 106:773-781.

7. Kruger, B., Lerch, M.M., and Tessenow, W. 1998. Direct detection of premature protease activation in living pancreatic acinar cells. Lab. Invest. 78:763-764.

8. Hofbauer, B., et al. 1998. Intra-acinar cell activa- tion of trypsinogen during caerulein-induced pancreatitis in rats. Am. J. Physiol. 275:G352-G362.

9. Wurmser, A.E., Gary, J.D., and Emr, S.D. 1999 Phosphoinositide 3-kinases and their FYVE domain-containing effectors as regulators of vac uolar/lysosomal membrane trafficking pathways. J. Biol. Chem. 274:9129-9132.

10. Simonsen, A., Wurmser, A.E., Emr, S.D., and Stenmark, H. 2001. The role of phosphoinositides in membrane transport. Curr. Opin. Cell Biol. 13:485-492.

11. Davidson, H.W. 1995. Wortmannin causes mistargeting of procathepsin D. Evidence for the involvement of a phosphatidylinositol 3-kinase in vesicular transport to lysosomes. J. Cell Biol. 130:797-805.

12. Bragado, M.J., Groblewski, G.E., and Williams, J.A. 1998. Regulation of protein synthesis by cholecystokinin in rat pancreatic acini involves PHAS-I and the p70 S6 kinase pathway. Gastroenterology. 115:733-742.

13. Nozu, F., Owyang, C., and Tsunoda, Y. 2000 Involvement of phosphoinositide 3-kinase and its association with pp60src in cholecystokininstimulated pancreatic acinar cells. Eur. J. Cell Biol. 79:803-809.

14. Rivard, N., Rydzewska, G., Lods, J.S., Martinez, J., and Morisset, J. 1994. Pancreas growth, tyrosine kinase, PtdIns 3-kinase, and PLD involve highaffinity CCK-receptor occupation. Am. J. Physiol. 266:G62-G70

15. Grady, T., et al. 1998. Zymogen proteolysis within the pancreatic acinar cell is associated with cellular injury. Am. J. Physiol. 275:G1010-G1017.

16. Gukovsky, I., Gukovskaya, A.S., Blinman, T.A., Zaninovic, V., and Pandol, S.J. 1998. Early NF-kappaB activation is associated with hormone-induced pancreatitis. Am. J. Physiol. 275:G1402-G1414.

17. Grady, T., Liang, P., Ernst, S.A., and Logsdon, C.D. 1997. Chemokine gene expression in rat pancreatic acinar cells is an early event associated with acute pancreatitis. Gastroenterology. 113:1966-1975.

18. Steinle, A.U., Weidenbach, H., Wagner, M., Adler, G., and Schmid, R.M. 1999. NF-kappaB/Rel activation in cerulein pancreatitis. Gastroenterology. 116:420-430.

19. Han, B., and Logsdon, C.D. 1999. Cholecystokinin induction of mob- 1 chemokine expres sion in pancreatic acinar cells requires NF-kap paB activation. Am. J. Physiol. 277:C74-C82.

20. Wymann, M.P., Sozzani, S., Altruda, F., Mantovani, A., and Hirsch, E. 2000. Lipids on the move: phosphoinositide 3-kinases in leukocyte function. Immunol. Today. 21:260-264.

21. Young, L.H., Ikeda, Y., Scalia, R., and Lefer, A.M 2000. Wortmannin, a potent antineutrophil agent, exerts cardioprotective effects in myocardial ischemia/reperfusion. J. Pharmacol. Exp. Ther. 295:37-43.

22. Gerszten, R.E., et al. 2001. Role of phosphoinositide 3-kinase in monocyte recruitment under flow conditions. J. Biol. Chem. 276:26846-26851.

23. Naccache, P.H., et al. 2000. Stimulation of human neutrophils by chemotactic factors is associated with the activation of phosphatidylinositol 3-kinase gamma. J. Biol. Chem. 275:23636-23641.

24. Sandoval, D., et al. 1996. The role of neutrophils and platelet-activating factor in mediating experimental pancreatitis. Gastroenterology. 111:1081-1091.

25. Bhatia, M., et al. 1998. The effects of neutrophil depletion on a completely noninvasive model of acute pancreatitis-associated lung injury. Int. J. Pancreatol. 24:77-83. 